\title{
Flujos de trabajo para el periodismo postindustrial: métodos y programas para una comunicación organizacional más ágil y transversal
}

\section{Workflows for the post-industrial journalism: Methods and tools to promote a more agile and transversal organizational communication}

\author{
José-María Valero-Pastor; Miguel Carvajal-Prieto; José-Alberto García-Avilés
}

Cómo citar este artículo:

Valero-Pastor, José-María; Carvajal-Prieto, Miguel; García-Avilés, José-Alberto (2019). “Flujos de trabajo para el periodismo postindustrial: métodos y programas para una comunicación organizacional más ágil y transversal". El profesional de la información, v. 28, n. 5, e280514.

https://doi.org/10.3145/epi.2019.sep.14

Artículo recibido el 09-04-2019

Aceptación definitiva: 11-06-2019

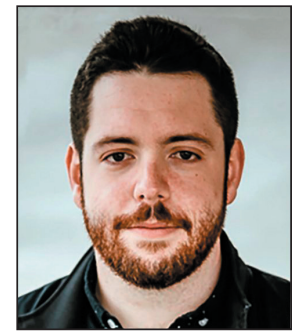

José-María Valero-Pastor https://orcid.org/0000-0002-6210-2761

Universidad Miguel Hernández de Elche Av. de la Universidad de Elche, s/n. 03202 Elche (Alicante), España jose.valerop@umh.es

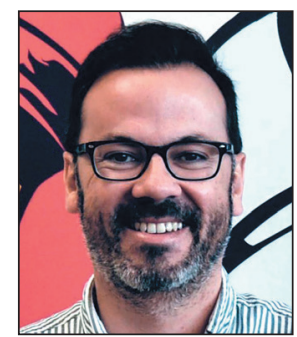

Miguel Carvajal-Prieto

https://orcid.org/0000-0001-6547-6171

Universidad Miguel Hernández de Elche Av. de la Universidad de Elche, $\mathrm{s} / \mathrm{n}$. 03202 Elche (Alicante), España mcarvajal@umh.es

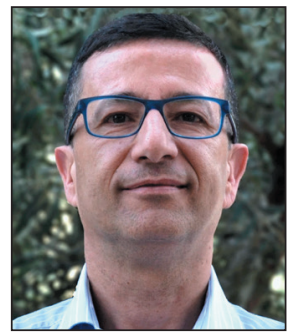

José-Alberto García-Avilés

https://orcid.org/0000-0001-7854-3476

Universidad Miguel Hernández de Elche Av. de la Universidad de Elche, $\mathrm{s} / \mathrm{n}$. 03202 Elche (Alicante), España Jose.garciaa@umh.es

\section{Resumen}

Los medios de comunicación buscan la supervivencia en un escenario caracterizado por los cambios constantes y acusados. Para reducir la incertidumbre y adaptarse al entorno, algunos optan por introducir flujos de trabajo flexibles en sus redacciones. En este trabajo se propone un modelo conceptual que identifica dos grupos de prácticas: 1) los métodos de ideación y creación de productos, que permiten el lanzamiento al mercado de activos que evolucionan continuamente para satisfacer las necesidades cambiantes de los usuarios; y 2) las metodologías ágiles para la gestión del trabajo cotidiano, que permiten una comunicación organizacional más fluida y transversal y, con ello, una mejor coordinación entre los profesionales y los equipos. Además, se aportan ejemplos concretos de cada tipo de flujo de trabajo, se estudia la relación entre ambos y se enumera una serie de aplicaciones tecnológicas que facilitan la flexibilidad y la comunicación en estos procesos.

\section{Palabras clave}

Medios de comunicación; Innovación mediática; Innovación organizacional; Comunicación organizacional; Flujos de trabajo; Metodologías ágiles; Gestión de proyectos; Design thinking; Lean startup; Scrum; Programación XP; Crystal; Kanban; Scrumban. 


\begin{abstract}
Media seek to survive in a scenario of constant and dramatic changes. News organizations introduce flexible workflows in their newsrooms to reduce uncertainty and to adapt to the environment. In this paper, we propose a conceptual model that identifies two groups of practices: 1) methods for designing and creating products, which allow to launch assets that continually evolve to meet the changing needs of users; and 2), agile methodologies to manage the daily work, which allow a more fluid and transversal organizational communication and, consequently, a better coordination between professionals and teams. In addition, we provide a specific set of examples of each type of workflow, and we also explore the relationship between both. Finally, we collect a varied range of technological tools that facilitate flexibility and communication.
\end{abstract}

\title{
Keywords
}

Media; Media innovation; Organizational innovation; Organizational communication; Workflows; Agile methodologies; Project management; Design thinking; Lean startup; Scrum; Extreme programming; Crystal; Kanban; Scrumban.

\section{Presentación}

La industria mediática se encuentra en un proceso de constante transformación cada vez más heterogénea y acusada, que afecta a la producción de contenidos y al lanzamiento de nuevos productos. Para reducir la incertidumbre las organizaciones periodísticas introducen flujos de trabajo más flexibles que facilitan su adaptación al entorno del periodismo postindustrial (Anderson; Bell; Shirky, 2012, p. 71). Esto fomenta la difusión de la innovación dentro de la entidad, mediante la comunicación organizacional y la transferencia de conocimiento (García-Avilés et al., 2018, p. 6).

La bibliografía especializada ya subrayó a principios de siglo la necesidad de modificar los flujos de trabajo para adaptarse a los procesos incipientes de convergencia mediática y digitalización (Verweij, 2009, p. 76), con iniciativas como la creación de una mesa de noticias única y la formación de grupos de trabajo multidisciplinares capaces de producir contenidos para múltiples canales. En definitiva, una mejor comunicación interna, que en la actualidad se potencia mediante la aplicación de metodologías ágiles, como Scrum o Kanban, y el uso de programas como Slack o Trello.

La aparición de móviles y redes sociales, con el consecuente aumento de la competencia en la economía de la atención, incentivaron el lanzamiento de nuevos productos y canales en esas plataformas. Los flujos de trabajo para la creación de estos activos debían ser flexibles, siguiendo las tendencias de la industria pujante del momento, el software (Dingsøyr et al., 2012). The New York Times, por ejemplo, emplea design thinking para renovar la página inicial de su sitio, y El confidencial para concebir nuevos productos como su aplicación móvil.

En este contexto la transformación de los flujos de trabajo emerge como un campo de estudio relevante y novedoso en el periodismo. Este estudio exploratorio identifica métodos y softwares que se introducen en las organizaciones periodísticas más innovadoras para fomentar una comunicación organizacional más ágil y transversal, que responda a los retos del ecosistema. Para ello se ha realizado una revisión bibliográfica que busca sintetizar las teorías y las prácticas más aceptadas sobre los flujos de trabajo en ámbitos ajenos al periodismo, que se están empezando a aplicar en la industria de los medios. Se propone un modelo conceptual que distingue entre los métodos de ideación y creación de nuevos productos y las metodologías ágiles para la coordinación del trabajo diario, aunque reconoce su estrecha vinculación como dos fases de un mismo proceso.

\section{Flujos de trabajo}

Se definen como la configuración de los aspectos relacionados con la labor cotidiana de la compañía: quién lleva a cabo cada acción, en qué orden y de qué manera, cómo se sincronizan los trabajadores, cómo fluye la información entre ellos y cómo se evalúa el cumplimiento de los objetivos (Yukl, 2013, p. 193). En los medios el concepto se ha referido tradicionalmente a las dinámicas de producción editorial, desde la asignación de historias hasta su distribución (García-Avilés et al., 2004).

Sin embargo en los últimos años los cambios en el proceso editorial han situado los flujos de trabajo como un tema central de la investigación académica en periodismo (Boczkowski; Mitchelstein, 2017). El mayor protagonismo de los usuarios, la aceleración de los ritmos informativos y el cambio de los ciclos de producción - por el cual los contenidos pueden ser completados, alterados y reusados constantemente- obligan a las compañías a repensar y flexibilizar sus flujos de trabajo (Anderson; Bell; Shirky, 2012, p. 60). 


\section{Flujos de trabajo para creación de productos: design thinking y lean startup}

Dos de los métodos de trabajo flexibles más destacados para la creación de nuevos productos son design thinking y lean startup (Carvajal, 2015; Maurya, 2012):

- design thinking: alude a la creatividad, y consiste en la comprensión de los problemas de los usuarios potenciales para ofrecerles una solución;

- lean startup: se centra en el testeo de un mínimo producto viable que irá creciendo o pivotando según la respuesta de los consumidores, ahorrando así tiempo y dinero.

Ambos métodos son compatibles, pues el primero puede entenderse como una fase previa del segundo (Hildenbrand; Meyer, 2012). En España, tanto startups periodísticas (Valero-Pastor; González-Alba, 2018) como grandes medios digitales como El confidencial, emplean estos métodos para generar sus productos.

\section{El design thinking busca soluciones inno- vadoras a problemas complejos, incor- porando al proceso las preocupaciones e intereses de las personas y huyendo de las rutas de pensamiento establecidas}

El design thinking busca soluciones innovadoras a problemas complejos, incorporando al proceso las preocupaciones e intereses de las personas y huyendo de las rutas de pensamiento establecidas. En él se recorren cuatro estados mentales (Carvajal, 2015, p. 52):

- pensamiento divergente, para generar alternativas a la realidad conocida y aportar nuevas elecciones;

- pensamiento convergente, para elegir las mejores opciones;

- pensamiento analítico, para descomponer los problemas en pequeños items;

- pensamiento sintético, para identificar los patrones de comportamiento.

Y luego se realiza un proceso de 5 fases:

- 1á: empatizar con los usuarios para entender sus necesidades.

- 2a: sintetizar la información recogida anteriormente para aislar y definir el problema.

- 3a: mediante una tormenta de ideas abierta al grupo de trabajo multidisciplinar, idear un producto potencial que cubra las necesidades.

- 4aa: prototipado, materializar la idea mediante esquemas visuales, así como evaluar si es posible ejecutarla. Para ello se debe situar entre los límites de lo realizable, lo viable y lo deseable, de tal manera que resulte barato y rápido, ya que lo más probable es que haya que modificarlo a corto plazo.

- 5a: testeo del prototipo en forma de mínimo producto viable, fase que conecta con el método lean startup.

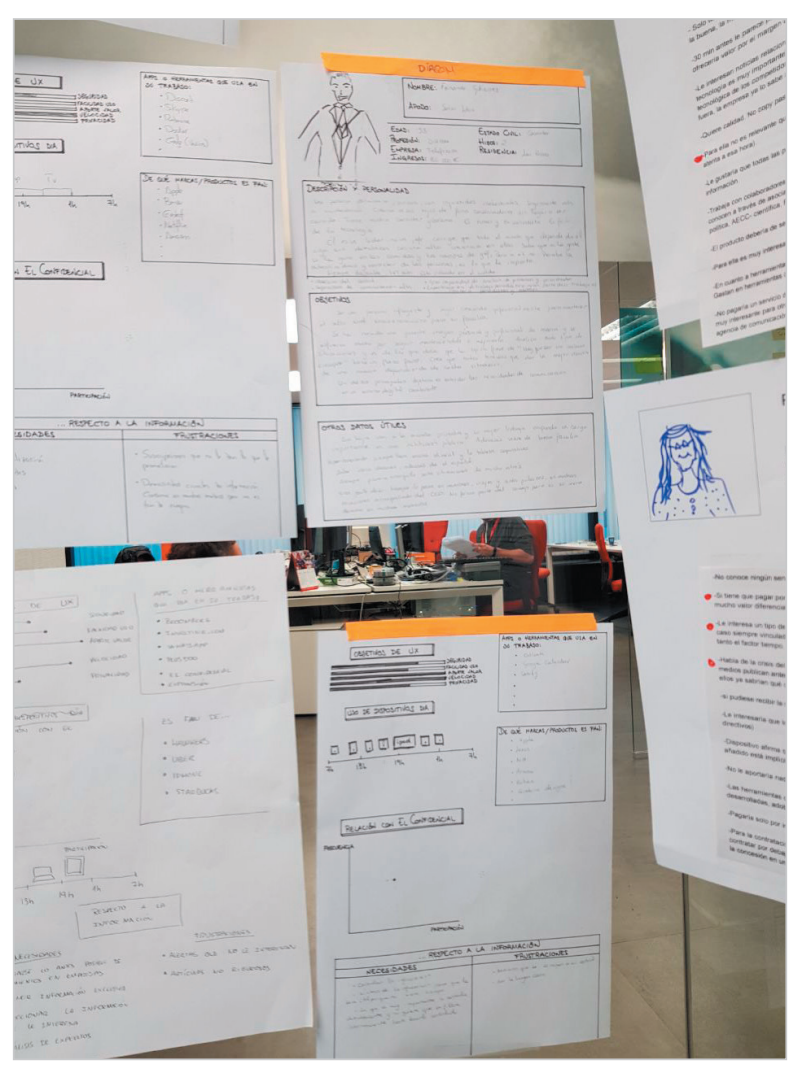

Figura 1. Documentación generada durante el proceso de design thinking en El confidencial lab

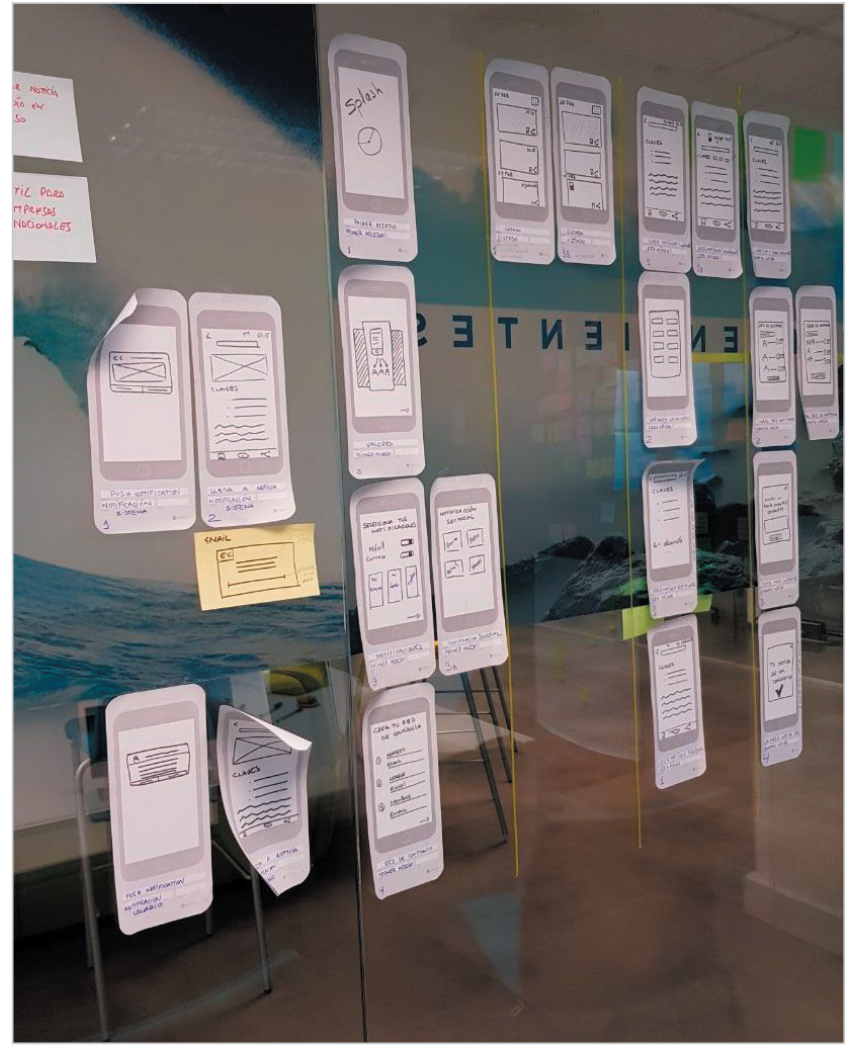

Figura 2. Prototipado de una aplicación móvil en El confidencial lab 
El lean startup contribuye a la creación y al lanzamiento de productos gracias a un proceso de validación ágil que aprende de la respuesta de los usuarios. El carácter cíclico del proceso, compuesto por:

- elaboración de prototipos,

- medición de sus desempeños, y

- aprendizaje obtenido con ello,

incorpora a los flujos de trabajo la necesidad de medir la eficacia de la innovación. En resumen, este método sirve para evaluar la pertinencia de una idea innovadora para satisfacer las necesidades de los usuarios, y pivotar si no se cumplen los objetivos. La validación viene seguida por un proceso de escalamiento para que los prototipos crezcan y cumplan objetivos más ambiciosos (Carvajal, 2015, p. 53).

Este método agiliza el emprendimiento. Quienes lo siguen suelen emplear el lean canvas, una simplificación del business model canvas diseñado por Osterwalder y Pigneur en 2009. El método consiste en plasmar una idea de producto de forma sencilla en un lienzo con nueve espacios: problema, solución, propuesta de valor, ventaja competitiva, segmento de clientes, métricas clave, canales, estructura de costes y flujo de ingresos (Maurya, 2012). Se sustituyen los extensos planes de negocio tradicionales por unos recursos fáciles de diseñar y susceptibles de mutar si el contexto lo requiere (Carvajal, 2015, p. 54).

\section{Metodologías ágiles para la organización del trabajo cotidiano}

Las metodologías ágiles transfieren los principios de flexibilidad y usabilidad inmediata al trabajo cotidiano, de la misma manera que el design thinking y el lean startup lo hacen con la creación de productos (Hildenbrand; Meyer, 2012; Schwaber; Sutherland, 2017). Este tipo de flujos de trabajo se originaron a principios de siglo para la ingeniería de software (Dingsøyr et al., 2012), una industria igual de cambiante que la mediática. Por ello su principal diferencia con las metodologías tradicionales es su carácter adaptativo, no predictivo, con respecto a los patrones de consumo y el entorno (Navarro-Cadavid; Fernández-Martínez; Morales-Vélez, 2013, p. 30).

Estos métodos han proliferado desde 2001, cuando se publicó el Manifiesto ágil, elaborado por la Agile Alliance. Este grupo de expertos estadounidenses pretendía

"esbozar los valores y principios que deberían permitir a los equipos desarrollar software rápidamente y respondiendo a los cambios que puedan surgir a lo largo del proyecto" (Letelier-Torres; Sánchez-López, 2003, p. 2).

El documento sostiene que los individuos y las interacciones prevalecen a los procesos y herramientas. Es más importante crear un buen equipo que empezar por el entorno y obligar a los profesionales a adaptarse a él. El manifiesto huye de los laberintos contractuales y la planificación cronificada, y ensalza la capacidad de respuesta (Beck et al., 2001; Letelier-Torres; Sánchez-López, 2003, p. 2; Letelier-Torres; Penadés, 2006, p. 3).

Basándonos en sus principios y máximas, se definen las metodologías ágiles como flujos de trabajo prácticos, adaptativos, flexibles y centrados en las personas, que se ejecutan en equipos de trabajo en constante comunicación entre sí y con el cliente, con el objetivo de entregarle constantemente partes del proyecto con valor añadido propio.

\subsection{Scrum}

Es uno de los métodos más populares (Green, 2016; Schwaber; Sutherland, 2017). La primera referencia sobre esta metodología, cuyo nombre proviene del vocabulario del rugby, data de 1986, cuando Nonaka y Takeuchi definen un nuevo enfoque en la elaboración de productos, más flexible y adaptativo, a partir de la formación de equipos multidisciplinares que trabajen juntos desde el principio hasta el final del proceso. Sin embargo, el método fue completamente desarrollado y popularizado a partir de los trabajos de Schwaber y Sutherland en los años 90.

El método Scrum se define como

"un marco desde el que aproximarse a problemas complejos y adaptativos al mismo tiempo que, de manera productiva y creativa, se lanzan productos del más alto valor posible" (Schwaber; Sutherland, 2017, p. 3).

Este modelo impulsa la formación de equipos de trabajo autogestionados con un flujo de trabajo guiado por roles, eventos y reglas orientadas

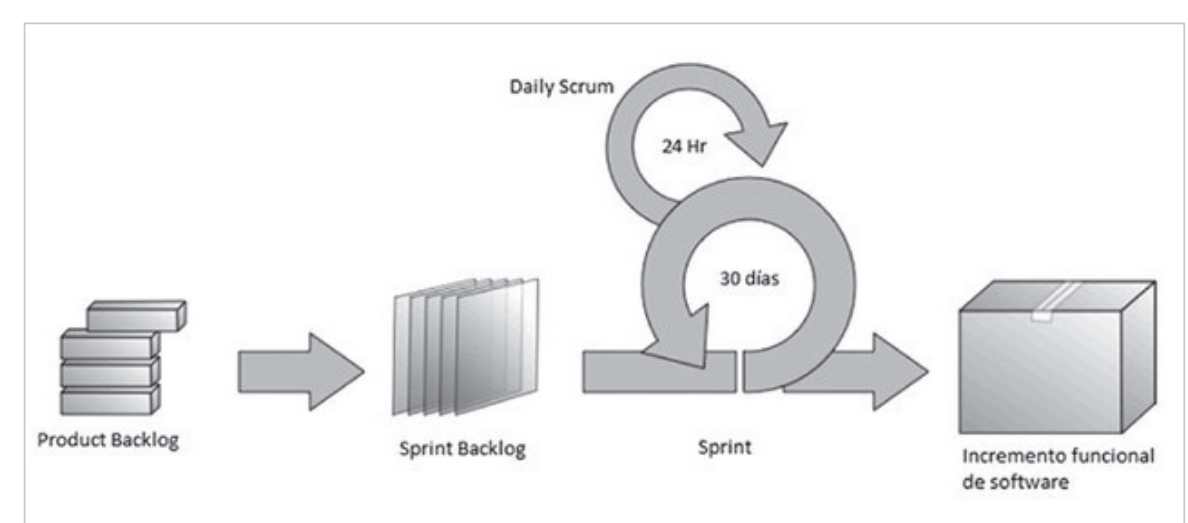

Figura 3. Proceso de producción en Scrum.

Fuente: Navarro-Cadavid; Fernández-Martínez; Morales-Vélez, 2013. 
a los objetivos generales (Navarro-Cadavid; Fernández-Martínez; Morales-Vélez, 2013, p. 33). Se asienta sobre los principios de (Green, 2016):

- transparencia entre los miembros del equipo;

- inspección, que permite detectar fallos en el proceso;

- adaptación, para modificar aspectos del proceso y el producto según los cambios del entorno.

Este método ágil divide la totalidad del trabajo que debe realizarse para la creación de un producto, conocido como product backlog, en pequeños conjuntos de tareas, el sprint backlog, que deben completarse en cada ciclo iterativo, que puede durar entre una semana y un mes y se denomina sprint (López-Menéndez, 2015, p. 8). El

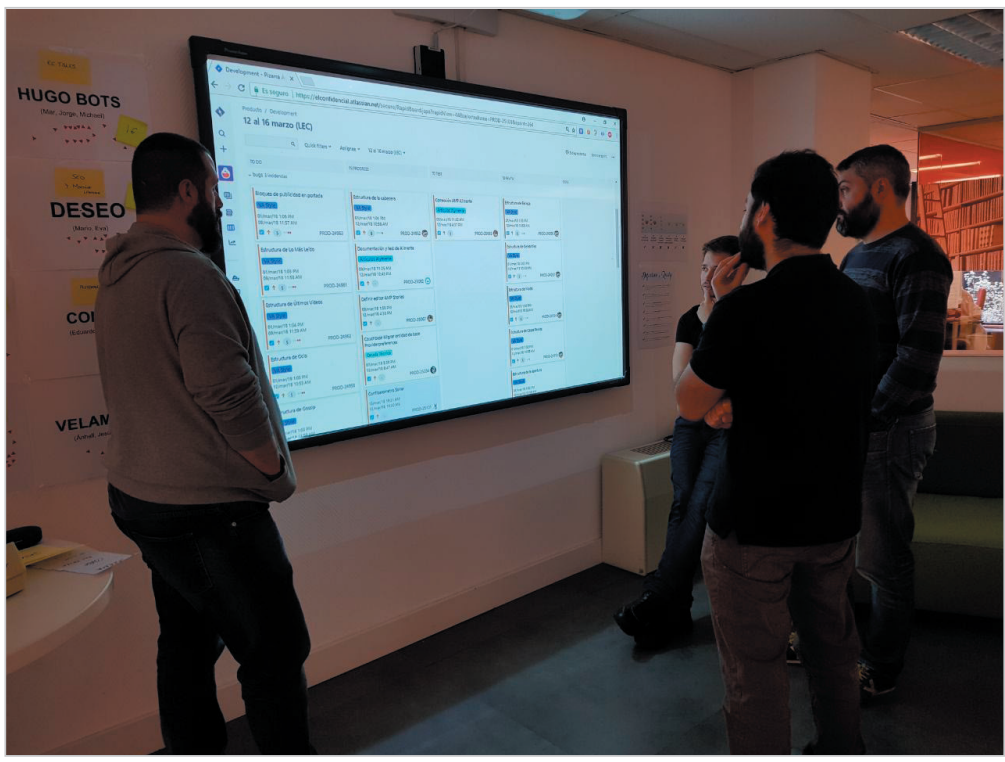

Figura 4. Daily scrum del equipo lector de El confidencial lab con la aplicación JIRA resultado de cada sprint debe ser un producto acabado y con el que el cliente pueda cubrir alguna necesidad (figura 3).

La metodología Scrum se caracteriza por la abundancia de reuniones entre los miembros del equipo (Letelier-Torres; Penadés, 2006, p. 6). Se proponen cuatro eventos (Green, 2016; Schwaber; Sutherland, 2017):

- planificación del sprint: en la que se decide qué tareas de la cola del producto se van a realizar en la siguiente iteración, teniendo en cuenta qué valor se puede aportar al producto en ese período;

- daily scrum: es una reunión diaria de 15 minutos en la que el equipo analiza el trabajo realizado el día anterior, planifica el del día actual e intenta identificar potenciales impedimentos;

- sprint review o evaluación del sprint: se lleva a cabo al final de cada iteración para analizar, junto con los stakeholders, lo que ya se ha hecho y los posibles cambios que se pueden añadir a la cola del producto;

- retrospectiva: sirve para que los equipos se analicen a sí mismos y detecten sus fortalezas y puntos débiles durante el pasado ciclo.

En cuanto a los roles profesionales, se necesita (Navarro-Cadavid; Fernández-Martínez; Morales-Vélez, 2013, p. 33):

- un Scrum master, un cargo supervisor que vele por la correcta aplicación de los valores, normas y prácticas del modelo;

- cada equipo debe estar capitaneado por un jefe de producto que represente a los stakeholders. Su labor es maximizar el valor del producto y coordinar el trabajo, estando entre sus competencias la gestión de la cola de producto;

- equipo de desarrollo que cuente con entre tres y nueve personas sin jerarquías entre ellas. Tienen la función de transformar lo que el cliente quiere en iteraciones funcionales del producto.

\subsection{Programación XP o extreme programming}

Fue concebido por Kent Beck, uno de los firmantes del Manifiesto ágil, para pequeños o medianos equipos inmersos en entornos cambiantes e inciertos (Beck, 1999; Beck; Andres, 2004). Sus valores elementales son la comunicación, la simplicidad, la retroalimentación, el coraje y el respeto (Beck; Andres, 2004, pp. 17-21), unas máximas que, según algunos investigadores, "son de sentido común pero llevadas al extremo, de ahí proviene su nombre" (Letelier-Torres; Sánchez-López, 2003, p. 4; Letelier-Torres; Penadés, 2006, p. 8).

Beck (1999) propone cinco principios para gobernar el flujo de trabajo:

- la retroalimentación rápida, de apenas minutos u horas;

- la asunción de la simplicidad, que permite ahorrar recursos en la mayoría de los casos;

- el cambio incremental, consistente en resolver los problemas mediante pequeñas modificaciones en los procesos y los productos ofrecidos;

- la apertura al cambio;

- el trabajo de calidad.

Además, añade algunos principios menos nucleares, como la responsabilidad aceptada, la comunicación abierta y honesta, la baja inversión inicial, y la medición de resultados.

Algunas prácticas recomendadas en el XP son:

- el planning game, que busca que el cliente exponga y ordene por prioridad las funciones del producto para unificar los criterios; 
- el cliente in situ, relacionado con lo anterior, que consiste en que los stakeholders se incorporen directamente a los flujos de trabajo;

- la programación por pares, para aumentar la creatividad y la transferencia de conocimiento.

Con la etiqueta de "proceso continuo", aparecen otras prácticas como (Beck, 1999; López-Menéndez, 2015, p. 9):

- las entregas pequeñas, que hace referencia a las iteraciones cortas tras las que se entrega al cliente un software terminado;

- la integración continua de las mejoras a los productos;

- la refactorización, que supedita la introducción de cambios en el software a que éste siga funcionando correctamente, para lo que se realizan pruebas unitarias.

Las prácticas que tienen que ver con el "entendimiento compartido" son el diseño simple, la metáfora, la propiedad colectiva del código y los estándares de programación (López-Menéndez, 2015: 9):

- el diseño simple: indica que los desarrolladores deben centrarse en responder a las necesidades de usuario planeadas para la presente iteración, y no en las posibilidades de crecimiento a largo plazo;

- la metáfora permite a los clientes y los desarrolladores compartir un lenguaje y, con ello, una forma de entender el trabajo;

- la propiedad colectiva del código permite a todos los desarrolladores modificar el proyecto, siempre que estén en disposición de añadirle valor, siguiendo unos estándares preestablecidos desde el inicio del proceso (Beck, 1999; Letelier-Torres; Penadés, 2006; Sampaio; Vasconcelos; Sampaio, 2004).

Por último, la programación XP también se preocupa por el "bienestar del programador" (López-Menéndez, 2015, p. 9). Por ello, recomienda la reducción del trabajo a 40 horas semanales para aumentar así la productividad (Beck, 1999; Letelier-Torres; Penadés, 2006; Sampaio; Vasconcelos; Sampaio, 2004). En una versión actualizada se añaden recomendaciones más prácticas para la aplicación de XP, que incluyen un espacio físico abierto y con paneles visuales, el carácter multidisciplinar e identitario de los equipos, y la construcción del software en 10 minutos ${ }^{1}$, entre otras (Beck; Andres, 2004).

Las fases de XP son la exploración, la planificación, las iteraciones, el productionizing y el mantenimiento (Navarro-Cadavid; Fernández-Martínez; Morales-Vélez, 2013: 35):

- exploración: se evalúa cuántas historias de usuario² deben formar parte de la primera entrega, teniendo en cuenta la funcionalidad del producto y el esfuerzo requerido;

- planificación: el cliente y los programadores definen las historias de usuario que se van a implementar y sus fechas;

- iteraciones: se implementan y prueban las historias de usuario completadas;

- productionizing: se afina el funcionamiento del programa y se despliega;

- mantenimiento: se continúa realizando mejoras.

Los principales roles son:

- cliente: debe ser parte activa del proceso para definir las funciones que debe tener el producto, los criterios de aceptación para considerar el proyecto exitoso y el presupuesto disponible;

- desarrollador: forma parte del equipo y se encarga de ejecutar las tareas que salen de las historias de usuario;

- tracker es un desarrollador que además se encarga de intentar optimizar todavía más los procesos, aunque es una figura prescindible;

- coach suele ser un consultor externo que ayuda a las empresas a implementar la metodología, y cuyo principal valor reside en que tiene experiencia acumulada en otras empresas.

\subsection{Crystal}

La familia de metodologías Crystal se caracteriza por su orientación a las personas en lugar de a los procesos, y la reducción al máximo de los elementos necesarios para llevarlos a cabo (Letelier-Torres; Sánchez-López, 2003, pp. 7; 36). Esta propuesta considera el desarrollo de software como un juego cooperativo de invención y comunicación, limitado por los recursos que se deben emplear. Su meta principal es entregar software útil, que funcione, y su objetivo secundario es preparar el siguiente juego; es decir, desarrollar nuevas funciones del producto creado u otros productos relacionados (Cockburn, 2001).

Sus principios se basan en la eliminación de tareas intermedias que puedan ralentizar las entregas de productos y la mejora de los procesos de comunicación organizacional. Se prioriza la cercanía física entre los profesionales, la proximidad de expertos y la seguridad personal para que cada trabajador pueda expresar sus inquietudes. Además se entiende que del mismo modo que cada proyecto es diferente y evoluciona también lo deben hacer las normas grupales, por lo que el refinamiento se hace sobre la marcha. Las dos reglas principales de Crystal dictan que se debe emplear el desarrollo incremental, siendo los ciclos inferiores a cuatro meses, y que el equipo debe mantener reuniones antes y tras cada incremento para afinar la metodología (Cockburn, 2001; Navarro-Cadavid; Fernández-Martínez; Morales-Vélez, 2013, p. 36). 
políticas de trabajo en equipo, que serán más rígidas cuanto más numerosos sean los grupos. Para ello se establece una clasificación por colores: desde Crystal Clear (3 a 8 miembros) hasta Crystal Red (50 a 100 miembros) (Letelier-Torres; Sánchez-López, 2003, p. 7).

\subsection{Kanban}

Fue introducida por Toyota para controlar el avance de los procesos de trabajo en el contexto de las líneas de producción. Su nombre es un vocablo japonés que se traduce como "tarjetas visuales" o "señales visuales", y su principal utilidad es la de controlar los inventarios y reducir los tiempos, por lo que se relaciona con la producción JIT (justo a tiempo) (Gamboa-Manzaba, 2014). A pesar de sus primeras aplicaciones a los procesos productivos mecánicos, actualmente también se emplea para la gestión de proyectos de software (Anderson, 2010).

Para Ordysinski (2013), esta técnica tiene como principales funciones:

- la visualización del flujo de trabajo;

- la determinación del límite de trabajo en curso en cada período;

- la medición del tiempo para completar cada tarea.

Kanban divide las tareas en partes, que se escriben en un post-it que incluye información como la fecha de entrega de la actividad, la fecha de inicio o la descripción. Estos post-its se colocan en tableros que tienen tantas columnas como estados o ciclos por los que avanza la tarea. De este modo todos los miembros del equipo visualizan qué trabajo se está realizando, quién lo realiza y cuáles son las prioridades en cada momento (Gamboa-Manzaba, 2014, p. 9).

Kanban establece que el número de tareas en curso debe acotarse previamente y permanecer inmutable a lo largo del proceso. Para poder agregar una nueva tarea al flujo, es necesario que otra se haya concluido. En cuanto a métricas, se establecen dos dimensiones: por un lado, el lead time, que cuenta el tiempo desde que se realiza una petición hasta que la tarea se entrega; y, por otro lado, el cycle time, que mide el lapso transcurrido entre el inicio de la tarea y su entrega (Anderson, 2010; Ordysínski, 2013). Otra característica del Kanban es la ausencia de roles preestablecidos (Gamboa-Manzaba, 2014).

\subsection{Scrumban}

La denominación, introducida por Ladas (2009), responde a la combinación de Scrum y Kanban. Empleando una metodología Scrum básica, los equipos trabajan en las tareas de la cola y, tras cada iteración, las mueven a la etapa de completadas de los tableros de Kanban. Con ello se puede visualizar el flujo de trabajo dentro y fuera de cada sprint e identificar los cuellos de botella, los recursos asignados a cada tarea y las prioridades establecidas (Ahmad-Khan, 2014; Gamboa-Manzaba, 2014; Ladas, 2009).

Las tareas son "empujadas hacia arriba" cuando es necesario, a diferencia del Scrum tradicional, en el que todas las tareas que van a ser realizadas en cada iteración deben ser previamente designadas para pasar del producto backlog al sprint backlog. Es decir, que la cola de tareas en Scrumban puede ser actualizada tantas veces como sea necesario a lo largo de una misma iteración (Ahmad-Khan, 2014, p. 33). Según Ladas (2009), sería aconsejable crear una cola intermedia entre la cola de producto y la de tareas a realizar. En ella se incluirían tareas que quedan pendientes en la reserva, pero que cuentan con una alta prioridad.

Otras características destacables de Scrumban son (Ahmad-Kahan, 2014, pp. 33-37; Gamboa-Manzaba, 2014, p. 11):

- limitación de las tareas llevadas a cabo en cada sprint, con el objetivo de que si algunos trabajadores o equipos acaban sus actividades designadas antes de lo previsto, no comiencen otras, sino que ayuden al resto a resolver los problemas que puedan surgir;

- reglas explícitas que ayudan a los equipos a autorregularse;

- reuniones de planificación más cortas para actualizar constantemente la cola del producto;

- utilización de estimaciones de esfuerzo para cada tarea. 
Tabla 1. Comparación entre los métodos Scrum, Kanban y Scrumban. Fuente: Ahmad-Khan, 2014 y Gamboa-Manzaba, 2014

\begin{tabular}{|l|l|l|l|}
\cline { 2 - 4 } \multicolumn{1}{c|}{ Scrum } & \multicolumn{1}{c|}{ Kanban } & Scrumban \\
\hline Visualización & Parcial & Total & Backlog con límites \\
\hline Backlogs & Sprint backlog, Product backlog & Backlog con límites & Limitado \\
\hline Límite del trabajo en proceso & No limitado & Limitado & Según sea necesario \\
\hline Cambios & $\begin{array}{l}\text { Debe esperar hasta el siguiente } \\
\text { sprint }\end{array}$ & Según sea necesario & Sí \\
\hline Roles & Sí & No & Sí \\
\hline Estimaciones & Sí & No & No \\
\hline Iteraciones & Sí & No & Depende de las decisiones de \\
\hline ScrumBoarda iteración
\end{tabular}

\section{Herramientas tecnológicas para la optimización de los flujos de trabajo}

La aplicación de las metodologías ágiles depende de las características de las empresas que las pretenden implementar. La tecnología disponible en las organizaciones - junto con otros factores como la estrategia empresarial, las cualidades de los profesionales y las peculiaridades del entorno- condiciona cómo se adoptan estas prácticas (Beck; Andres, 2004).

El sistema de gestión de contenidos (CMS) es un buen ejemplo. El CMS sostiene el flujo de trabajo en entornos de publicación digital y permite a los profesionales desarrollar más eficientemente sus prácticas y tareas de publicación. Verweij (2009, p. 81) por ejemplo, identifica el uso de un CMS común a toda la redacción como la condición para el éxito de los procesos de convergencia, pues garantiza la coordinación entre departamentos. El CMS mejora los flujos de trabajo, pues permite que más usuarios trabajen a la vez, de forma fluida y colaborativa, con controles de calidad y empleando un conocimiento compartido, creado y almacenado en la propia empresa (Robertson, 2003).

Además del CMS, existen otras aplicaciones para la gestión de proyectos que comulgan con las metodologías ágiles, como Trello, Asana, JIRA y Slack. Estos programas informáticos basados en esta filosofía se describen a continuación:

\section{Trello}

Es una aplicación web basada en Kanban que permite crear un tablero por cada proyecto. Los usuarios introducen listas de tareas que posteriormente dividen en subgrupos llamados tarjetas. Estas tarjetas, que se pueden arrastrar de una lista a otra, incluyen información como descripción de tareas, listas de pasos para completarlas, comentarios posteriores, documentos adjuntos, iniciales de los miembros del equipo a quienes se les ha asignado y la fecha de entrega, que se puede sincronizar con los calendarios de Outlook o Gmail de los trabajadores. Las tarjetas se pueden clasificar por etiquetas de color o categorías como "completado" o "en espera". Trello incluye la posibilidad de que la aplicación notifique cuando una tarjeta está inactiva por mucho tiempo.

\section{Asano}

Es similar a Trello, y también emplea el método Kanban. En España la usan medios donde gran parte de sus plantillas trabajan en remoto, como WeblogSL. También incluye tableros para la medición del progreso de proyectos, en los que se indican las tareas completadas y pendientes. La principal diferencia entre ambas aplicaciones, según Gould (2018, p. 3), son las oportunidades de colaboración. Mientras que Asana habilita más canales de comunicación con otros miembros del equipo dentro de la aplicación, Trello prioriza la compatibilidad con medios externos.

\section{JIRA}

Se diseñó para ayudar a los programadores a trabajar en equipo, colaborar y gestionar un proceso de creación de software, con tareas como la reparación de errores y la documentación. Las versiones posteriores y la creación de nuevos plugins le han añadido funciones como la incorporación de soporte para metodologías ágiles, convirtiéndose así en uno de los programas más sofisticados del mercado (Juzko; Tjakradiwiria, 2011, p. 20). Es utilizado por ejemplo, para la creación de productos en el laboratorio de El confidencial. Planner de Microsoft es similar y permite además la integración con productos de la suite de la compañía (Gould, 2018).

\section{Slack}

Tiene un carácter más comunicativo. Constituye a la vez una plataforma de trabajo digital y un sistema de gestión de la comunicación en grupos pequeños y medianos. Su principal objetivo es aumentar la productividad y mejorar la eficiencia de los equipos. Un espacio de trabajo en Slack comprende varios canales, que son conversaciones dedicadas a un proyecto específico, y puede adoptar di-
Estos métodos proveen a los equipos de trabajo de normas, roles y dinámicas que les permiten generar en cada iteración productos escalables pero con un valor añadido propio 
versas formas, dependiendo de las necesidades percibidas por los líderes de los equipos. Éstos también pueden controlar el acceso de los miembros a canales concretos, y los usuarios pueden abandonarlos o archivarlos cuando lo consideren oportuno, de manera que ahorran tiempo y atención (Johnson, 2018). En España medios como Verne, Maldita.es y eldiario.es emplean este programa para dinamizar sus flujos de trabajo y evitar la sobrecarga de reuniones.

\section{Conclusiones}

En este artículo se ha introducido un modelo conceptual que sintetiza herramientas y métodos que facilitan la adaptación de los flujos de trabajo de los medios en el contexto del periodismo post-industrial. Las organizaciones periodísticas han tenido que repensar y flexibilizar sus flujos de trabajo por la influencia de la digitalización, la integración de redacciones, el contenido generado por los usuarios, la aceleración de los ritmos informativos y el cambio en los ciclos de producción.

Entre los métodos que fomentan la transformación de los flujos de trabajo en el periodismo, hemos identificado, por un lado, los que ayudan en los procesos de ideación y creación de nuevos productos y, por otro, los que facilitan la coordinación del trabajo diario. En el primer grupo se hallan el design thinking y el lean startup, que constituyen dos procesos flexibles para la ideación y puesta en marcha de productos, respectivamente, aten-

Los métodos de ideación y creación de productos y las metodologías ágiles para la gestión del trabajo cotidiano comparten el objetivo de maximizar la satisfacción de necesidades a los clientes de forma continua diendo constantemente a las necesidades de los usuarios. En el segundo, métodos como Scrum, Crystal, Kanban y Scrumban son métodos de flujo de trabajo prácticos, adaptativos, flexibles y centrados en la comunicación interpersonal, que tienen como objetivo la entrega continua al cliente de partes del producto con valor añadido propio.

Además, se ha analizado la relación entre ambas categorías y se concluye que los métodos de ideación y creación de productos y las metodologías ágiles para la gestión del trabajo cotidiano comparten un mismo objetivo: maximizar la satisfacción de necesidades a los clientes de forma continua. Mediante su empleo las organizaciones incentivan una comunicación organizacional más ágil y transversal, que flexibiliza su estructura para responder constantemente a los retos del ecosistema con el menor coste y esfuerzo.

La propuesta de este marco conceptual establece que unas y otras constituyen diferentes niveles de un mismo proceso. En primer lugar, la fase del design thinking consiste en identificar problemas del público objetivo, idear productos para satisfacerlas y diseñar prototipos. Es ahí donde se inicia la fase lean startup, en la que se materializa el producto, se miden sus resultados con clientes reales y se decide si se debe pivotar o escalar para construir nuevas funciones. Las metodologías ágiles entran en juego en ese proceso de construcción, tanto del mínimo producto viable como de cada una de las iteraciones posteriores. Estos métodos proveen a los equipos de trabajo de normas, roles y dinámicas que les permiten generar en cada iteración productos escalables pero con un valor añadido propio.

Por último, el presente trabajo presenta algunas limitaciones que pueden subsanarse en futuras investigaciones. La propuesta conceptual exige la búsqueda de mayores evidencias empíricas que permitan evaluar el impacto de estos métodos de trabajo en las organizaciones periodísticas.
La fase del design thinking consiste en identificar problemas del público objetivo, idear productos para satisfacerlas y diseñar prototipos

\section{Notas}

1. Extreme Programming es una metodología extremadamente ágil que recomienda que toda la base de código de las nuevas funcionalidades pueda ser construida (y los tests funcionales conducidos) en menos de 10 minutos.

2. Historias de usuario son requisitos o funcionalidades que se incorporan a los productos en cada iteración. Se formulan desde el punto de vista de los usuarios para asegurarse de que cada una de ellas aporta valor añadido a la audiencia, pero en la práctica suponen tareas que los equipos deben cumplir cada semana. Suelen expresarse en un par de frases como máximo.

\section{Bibliografía}

Ahmad-Khan, Zahoor (2014). Scrumban-adaptive agile development process. Helsinki: Helsinki Metropolia University of Applied Sciences. https://www.theseus.fi/bitstream/handle/10024/77014/Khan_Zahoor.pdf?sequence=1

Anderson, David (2010). Kanban. Successful evolutionary change for your technology business. Sequim: Blue Hole Press. ISBN: 9780984521401 
Anderson, Chris W.; Bell, Emily J.; Shirky, Clay (2012). Post-industrial journalism: Adapting to the present. Columbia: Tow Center for Digital Journalism. https://doi.org/10.7916/D8N01JS7

Beck, Kent (1999). Extreme programming explained: Embrace change. Reading: Addison Wesley. ISBN: 9780201616415

Beck, Kent; Andres, Cynthia (2004). Extreme programming explained. Embrace change. Reading: Addison Wesley. ISBN: 0321278658

Beck, Kent; Beedle, Mike; Van-Bennekum, Arie; Cockburn, Alistair; Cunningham, Ward; Fowler, Martin; Highsmith, Jim; Hunt, Andrew; Jeffries, Ron; Kern, Jon; Marick, Brian; Martin, Robert C.; Schwaber, Ken; Sutherland, Jeff; Thomas, Dave (2001). The agile manifesto. http://agilemanifesto.org/iso/es/principles.html

Boczkowski, Pablo J.; Mitchelstein, Eugenia (2017). "Scholarship on online journalism: Roads traveled and pathways ahead". In: Boczkowski, Pablo J.; Anderson, Chris W. Remaking the news. Essays on the future of journalism scholarship in the digital age. Cambridge: MIT Press, pp. 15-26. ISBN: 9780262036092

Carvajal, Miguel (2015). "Lean startup y design thinking. Nuevos métodos para innovar en periodismo". En: Manfredi-Sánchez, Juan-Luis. Innovación y periodismo: emprender en la universidad. Laguna: CAC, pp. 47-56. ISBN: 978 84 15698937

http://www.revistalatinacs.org/067/cuadernos/2015/cac76.pdf

Cockburn, Alistair (2001). Agile software development. Reading: Addison-Wesley. ISBN: 9780201699692

Dingsøyr, Torgeir; Nerur, Sridhar; Balijepally, Venugopal; Moe, Nils-Brede (2012). "A decade of agile methodologies: Towards explaining agile software development". Journal of systems and software, v. 85, n. 6, pp. 1213-1221. https://doi.org/10.1016/j.jss.2012.02.033

Gamboa-Manzaba, Julio-César (2014). “Aumento de la productividad en la gestión de proyectos, utilizando una metodología ágil aplicada en una fábrica de software en la ciudad de Guayaquil”. Revista tecnológica Espol, v. 27, n. 2, pp. 1-36. http://www.rte.espol.edu.ec/index.php/tecnologica/article/view/312

García-Avilés, José-Alberto; Carvajal-Prieto, Miguel; Arias, Félix; De-Lara-González, Alicia (2018). “How journalists innovate in the newsroom. Proposing a model of the diffusion of innovations in media outlets". Journal of media innovations, v. 5, n. 1, pp. 1-16.

https://www.journals.uio.no/index.php/TJMI/article/view/3968

https://doi.org/10.5617/jomi.v5i1.3968

Gould, Elyssa M. (2018). "Workflow management tools for electronic resources management". Serials review, v. 44, n. 1, pp. 71-74.

https://doi.org/10.1080/00987913.2018.1434380

Green, David (2016). Scrum. Novice to ninja. EUA: SitePoint. ISBN: 9780994346919

Hildenbrand, Tobias; Meyer, Johannes (2012). "Intertwining lean and design thinking: Software product development from empathy to shipment". En: Maedche, Alexander; Achim Botzenhardt; Neer, Ludwig. Software for people. Management for professionals. Berlin: Springer, pp. 217-237. ISBN: 9783642313714 https://doi.org/10.1007/978-3-642-31371-4_13

Johnson, Heather A. (2018). Slack. Journal of the Medical Library Association, v. 106, n. 1, pp. 148-151. https://doi.org/10.5195/jmla.2018.315

Juzko, Michailas; Tjakradiwiria, Hermawan (2011). Project management tools in software development. Jönköping: Jönköping University.

Ladas, Corey (2009). Scrumban - Essays on kanban systems for lean software development. EUA: Modus Cooperandi Press. ISBN: 9780578002149

Letelier-Torres, Patricio; Penadés, Carmen (2006). “Metodologías ágiles para el desarrollo de software: eXtreme Programming (XP)". Técnica administrativa, v. 5, n. 26.

http://www.cyta.com.ar/ta0502/v5n2a1.htm

Letelier-Torres, Patricio; Sánchez-López, Emilio A. (eds.) (2003). Metodologías ágiles en el desarrollo de software. València: Universitat Politècnica de València.

http://issi.dsic.upv.es/archives/f-1069167248521/actas.pdf

López-Menéndez-De-Jiménez, Rina-Elizabeth (2015). “Metodologías ágiles de desarrollo de software aplicadas a la gestión de proyectos empresariales". Revista tecnológica, v. 8, pp. 6-11.

http://www.redicces.org.sv/jspui/bitstream/10972/2917/1/Articulo1.pdf 
Maurya, Ash (2012). Running lean: Iterate from Plan A to a Plan that works. Cambridge: O'Reilly. ISBN: 9781306797160

Navarro-Cadavid, Andrés; Fernández-Martínez, Juan-Daniel; Morales-Vélez, Jonathan (2013). "Revisión de metodologías ágiles para el desarrollo de software". Prospectiva, v. 11, n. 2, pp. 30-39.

https://www.redalyc.org/pdf/4962/496250736004.pdf

Ordysinski, Thomasz (2013). "Kanban based information management in organizations". In: Polish Association for Knowledge Management, pp. 76-85.

http://pszw.edu.pl/en/publications/item/1051-tomt063-4

Robertson, James (2013). "So, what is a CMS?". Step two, June 3.

https://www.steptwo.com.au/papers/kmc_what

Sampaio, Américo; Vasconcelos, Alexandre; Sampaio, Pedro R. Falcone (2004). "Assessing agile methods: An empirical study". Journal of the Brazilian Computer Society, v. 10, n. 2, pp. 21-48.

https://doi.org/10.1007/BF03192357

Schwaber, Ken; Sutherland, Jeff (2017). The Scrum guide.

https://www.scrumguides.org/docs/scrumguide/v2017/2017-Scrum-Guide-US.pdf

Valero-Pastor, José-María; González-Alba, José-Antonio (2018). “Las startups periodísticas como ejemplos de innovación en el mercado mediático español. Estudio de casos". Revista latina de comunicación social, v. 73, pp. 556-582.

https://doi.org/10.4185/RLCS-2018-1269

Verweij, Peter (2009). "Making convergence work in the newsroom: A case study of convergence of print, radio, television and online newsrooms at the African media matrix in South America during the National Arts Festival". Convergence: The international journal of research into new media technologies, v. 15, n. 1, pp. 75-87.

https://doi.org/10.1177/1354856508097020

Yukl, Gary (2013). Leadership in organizations. New Jersey: Pearson. ISBN: 9780132771863

https://www.academia.edu/14679950/Leadership_in_Organizations_Gary_Yu_K
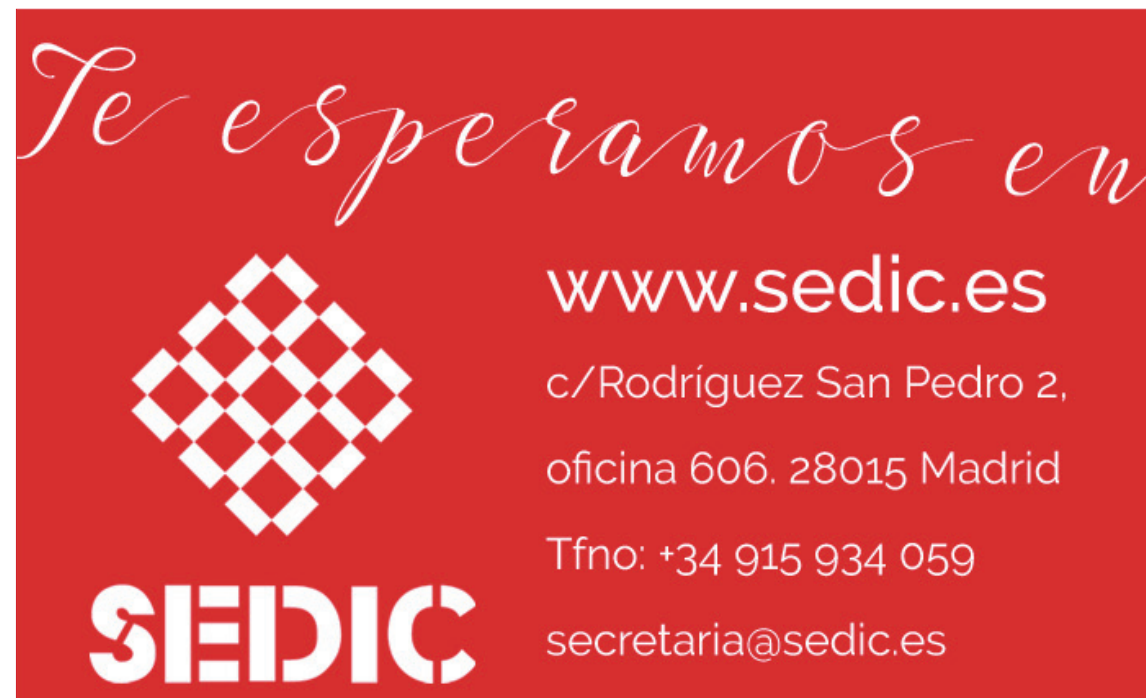

WWW.sedic.es

c/Rodríguez San Pedro 2,

oficina 606. 28015 Madrid

Tfno: +34915934059

secretaria@sedic.es

Sociedad

Española de

Documentación e Información

Científica

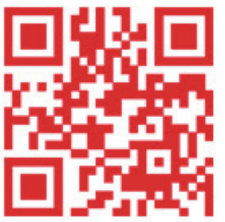

https://twitter.com/SEDIC2O

if https://wwww.facebook.com/AsociacionSEDIC

in https://www.linkedin.com/groups?home=\&gid=5060038 